\title{
Optimization of an Electromagnetic Energy Harvesting Device
}

\author{
Chitta Ranjan Saha ${ }^{1}$, Terence O’Donnell ${ }^{1}$, Heiko Loder $^{2}$, Steve Beeby ${ }^{3}$, and John Tudor ${ }^{3}$ \\ ${ }^{1}$ Tyndall National Institute, Cork, Ireland \\ ${ }^{2}$ Department of Electrical Engineering, University of Applied Science, Fachhochschule Gie $\beta$ en-Friedberg 61169, Germany \\ ${ }^{3}$ Department of Electrical and Computer Science, University of Southampton, Southampton 30171BJ, U.K.
}

This paper presents the modeling and optimization of an electromagnetic-based generator for generating power from ambient vibrations. Basic equations describing such generators are presented and the conditions for maximum power generation are described. Two-centimeter scale prototype generators, which consist of magnets suspended on a beam vibrating relative to a coil, have been built and tested. The measured power and modeled results are compared. It is shown that the experimental results confirm the optimization theory.

Index Terms-Displacement, energy conversion, generators and resonance.

\section{INTRODUCTION}

$\mathbf{E}$ NERGY harvesting techniques, where energy is generated from ambient vibrations in the environment have recently generated interest from the point of view of supplying "everlasting" power for remote sensor nodes. Vibrational power generators based on electromagnetic [1]-[4], piezoelectric [5], and electrostatic principles [6] have already been reported. Electromagnetic-based generators have been demonstrated to generate power levels ranging from 0.3 to $800 \mu \mathrm{W}$ from vibration frequencies of several hundred hertz. For example, El-hami et al. [3] showed that $0.53 \mathrm{~mW}$ could be generated from a $25-\mu \mathrm{m}$ amplitude vibration at $322-\mathrm{Hz}$ vibration using a 510-mg moving mass. Glynne-Jones et al. [4] demonstrated an electromagnetic generator which was capable of generating $157 \mu \mathrm{W}$, when placed in a car engine compartment. Williams et al. [2] described an electromechanical model for electromagnetic generators and derived the optimum damping conditions, however, due to low electromagnetic damping, the optimum condition could not be verified in practice. Mitcheson et al. [6] described a general theoretical analysis for vibration driven generators, including both electromagnetic and electrostatic approaches, however, no comparison between the models and measurements were presented. In previous work, the authors have presented the structure, design [7], and fabrication with initial test results [8] for a micromachined electromagneticbased vibration generator. However, it proved difficult to verify the optimization theory on these devices due to their size and structure.

In this paper, the model for an electromagnetic-based, vibrational power generator is presented and the model is used to investigate the optimum conditions for electromagnetic damping and load resistance. Furthermore, the optimum conditions have been verified using measurements on two macrogenerators which have been built and tested. The measured and predicted results for the two generators are compared.

Digital Object Identifier 10.1109/TMAG.2006.879447

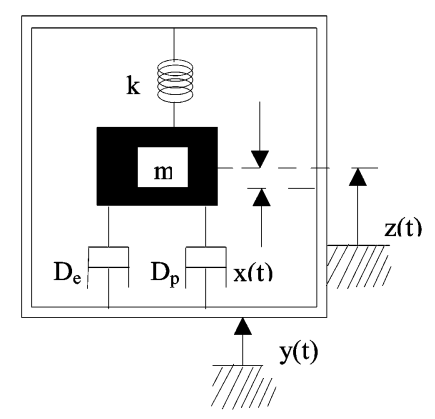

Fig. 1. Schematic diagram of electromagnetic generator.

The eventual aim of this work is to use the verified models presented here to target improvements in the performance of the micromachined generators.

\section{GENERATOR MODEL}

The basic vibrational energy generator consists of a mass mounted on a spring which vibrates relative to housing when subjected to an external vibrational force. The mechanical energy of the moving mass is transformed to electrical energy by having the mass move a magnet relative to a coil. The mass may consist of the magnet itself or the coil. It has been suggested that the motion of such a system can be reasonably well-described by a second-order mass $(m)$-spring $(k)$-damper $\left(D_{p}\right)$ system, as shown in Fig. 1. The basic equation of motion of the mass relative to the housing when driven by a sinusoidal vibration force, $F(=m a$, where $a$ is the acceleration), is given by [2]

$$
m \frac{d^{2} x}{\mathrm{dt}^{2}}+D_{p} \frac{\mathrm{dx}}{\mathrm{dt}}+\mathrm{kx}=F_{o} \sin \omega t-F_{\mathrm{em}}
$$

where $x$ is the relative movement between mass and housing (or between magnet and coil), $D_{p}$ is the parasitic damping force due to air resistance and material loss, and $F_{\text {em }}$ is the electromagnetic force due to the force between the current in the coil and magnets. This system will have a mechanical resonant frequency, $\omega_{n}$, where $\omega_{n}=\sqrt{k / m}$ and, in general, it is desirable 
to have the frequency of the driving force equal to the mechanical resonant frequency in order to maximize displacement.

The electromagnetic damping force is proportional to the velocity [6] and can be expressed as

$$
F_{\mathrm{em}}=\left(N \frac{d \varphi}{\mathrm{dx}}\right)^{2} \frac{1}{R_{c}+R_{l}+j \omega L} \frac{\mathrm{dx}}{\mathrm{dt}}=D_{\mathrm{em}} \frac{\mathrm{dx}}{\mathrm{dt}}
$$

where $D_{\mathrm{em}}$ is the electromagnetic damping. The solution of (2) at the resonance condition $\left(\omega=\omega_{n}\right)$ defines the displacement, and is given by

$$
x=-F_{o} \cos \omega t /\left[\left(D_{p}+D_{\mathrm{em}}\right) \omega\right]
$$

The average generated electrical power can be obtained from

$$
P_{\mathrm{avg}}=\frac{1}{T} \int_{0}^{T} D_{\mathrm{em}}\left(\frac{\mathrm{dx}}{\mathrm{dt}}\right)^{2} \mathrm{dt}=D_{\mathrm{em}} \frac{F_{o}^{2}}{2\left(D_{p}+D_{\mathrm{em}}\right)^{2}}
$$

By differentiating $P_{\text {avg }}$ with respect to $D_{\text {em }}$ and equating to zero, it can be shown that the maximum electrical power occurs when $D_{\mathrm{em}}=D_{p}$. Therefore, the objective of the generator design should be to achieve this optimum electromagnetic damping, i.e., it should aim to make electromagnetic damping equal to the parasitic damping. As can be seen from (2), the electromagnetic damping can be varied by changing the load resistance, coil impedance, and magnet flux. For frequencies of less than $1 \mathrm{kHz}$, we assume that the inductive impedance of the coil can be neglected with respect to the resistance. By assuming that $D_{\mathrm{em}}=D_{p}$ and using (2) the optimum load resistance given by

$$
R_{\mathrm{optl}}=\frac{1}{D_{p}} N^{2}\left(\frac{d \phi}{\mathrm{dx}}\right)^{2}-R_{c}
$$

However, the coil resistance $R_{c}$ depends on the number of turns $N$. The relationship between the coil resistance and the number of turns depends on the coil technology. For a fixed size wire wound multilayer coil, the coil resistance can be shown to depend on the square of $N$, i.e., $R_{c}=k N^{2}$ [9], where $k$ is a proportionality constant. Thus, the optimum load resistance would be

$$
R_{\mathrm{optl}}=\left(\frac{1}{D_{p}}\left(\frac{d \phi}{\mathrm{dx}}\right)^{2}-k\right) N^{2}
$$

We can see from (6) that the optimum load resistance can only be found (i.e., positive resistance) if $k$ is less than the first term within the brackets in (6). Thus, if parasitic damping is very high or if the flux gradient is low, the situation can arise where the optimum load resistance cannot be found. This occurs when the electromagnetic damping is low and cannot be increased to equal the parasitic damping.

If electromagnetic damping is very low compared to parasitic damping $\left(D_{p}>>D_{\mathrm{em}}\right)$, then the displacement given by (3) is determined by the parasitic damping. In this case, the load power is simply obtained from the voltage division between load resistance and coil resistance and the maximum power will be transferred to the load when the load and coil impedance are equal.

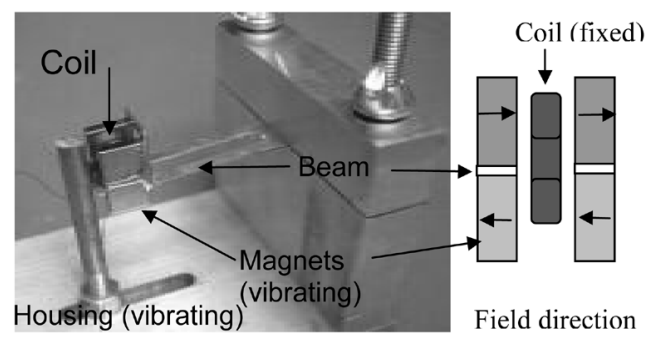

Fig. 2. Image of generator A showing the magnets attached to a copper beam and wire-wound coil.

TABLE I

GENERATOR PARAMETERS

\begin{tabular}{|l|l|l|}
\hline Parameters & Generator-A & Generator-B \\
\hline Moving mass $(\mathrm{kg})$ & 0.0428 & 0.025 \\
\hline Magnet size $(\mathrm{mm})$ & $15 \times 15 \times 5$ & $10 \times 10 \times 3$ \\
\hline Beam frequency $(\mathrm{Hz})$ & 13.11 & 84 \\
\hline Acceleration $\left(\mathrm{m} / \mathrm{s}^{2}\right)$ & 0.78 & 7.8 \\
\hline Magnet and coil gap $(\mathrm{mm})$ & 1.25 & 1.5 \\
\hline Coil outer diameter $(\mathrm{mm})$ & 28.5 & 13.3 \\
\hline Coil inner diameter $(\mathrm{mm})$ & 5 & 2 \\
\hline Coil thickness $(\mathrm{mm})$ & 7.5 & 7 \\
\hline Coil turns & 850 & 300 \\
\hline Coil resistance $(\mathrm{ohm})$ & 18 & 3.65 \\
\hline
\end{tabular}

\section{RESUlts}

Two electromagnetic generators were built and vibrated using a controllable force by an electromagnetic shaker. These consisted of four magnets (NdFeB35) as shown in Fig. 2, arranged to create a high flux gradient and attached to a copper beam. A wire-wound copper coil was placed between the magnets. This generator construction is based on the type of generator described in [7]. Table I gives the coil and magnet parameters for generators $\mathrm{A}$ and $\mathrm{B}$. The generators were vibrated using a sinusoidal acceleration with the frequency matched to the generator mechanical resonant frequency. The displacement and voltage were measured for various load resistances. The load power is calculated from the voltage and load resistance. The displacement at no-load (open circuit) can be used in (3), with $D_{\mathrm{em}}=0$ to estimate the parasitic damping. The generators were also simulated using a three-dimensional (3-D) finite element (FE) transient model with the measured displacement as input. The flux gradient can be obtained from the FE model and used in (2) to calculate the electromagnetic damping. The parasitic and electromagnetic damping can then be used in (4) to calculate the power.

Figs. 3 and 4 show the measured displacement and the measured and modeled load voltage for different load conditions for generators A and B, respectively. The measured and modeled voltages agree quite closely. Figs. 5 and 6 show the measured and calculated power, and the estimated parasitic and electromagnetic damping, for generators A and B, respectively.

The graph in Fig. 3 shows that there is a significant change in displacement with the change in load, for generator A, however, for generator B the displacement does not vary with load. This is consistent with the fact that for generator A the electromagnetic damping is comparable to the parasitic damping, but for generator $\mathrm{B}$ the electromagnetic damping is much lower than the 


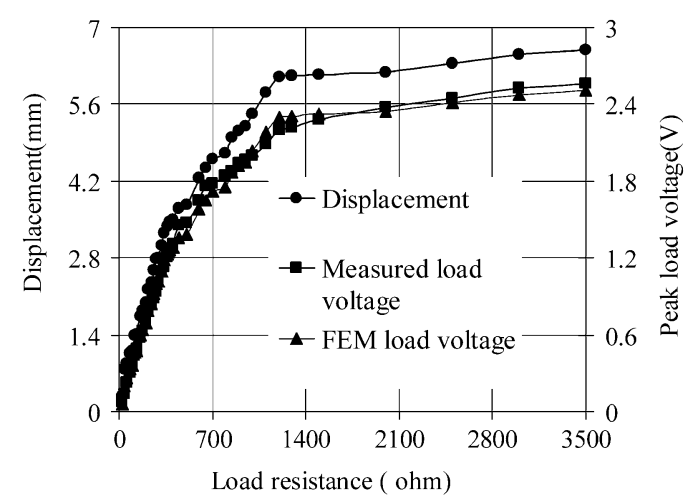

Fig. 3. Measured displacement and modeled and measured load voltage for generator $\mathrm{A}$.

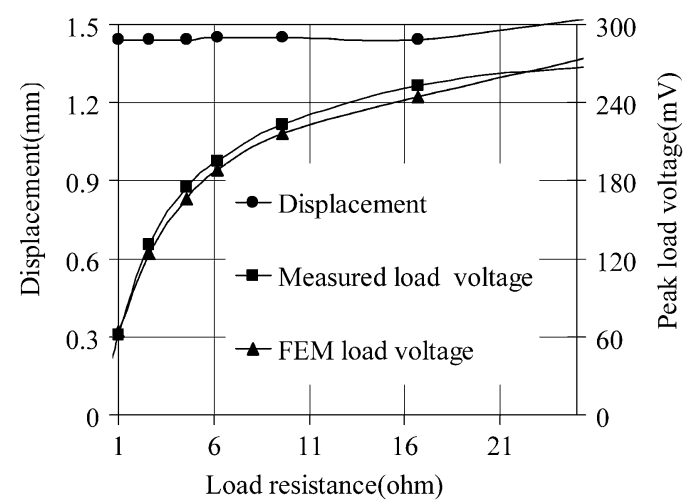

Fig. 4. Measured displacement and modeled and measured load voltage for generator B.

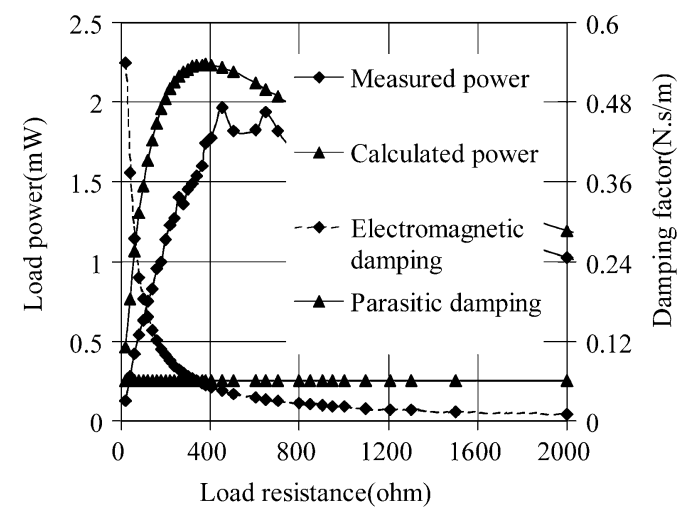

Fig. 5. Measured and calculated load power and estimated parasitic and electromagnetic damping for generator A.

parasitic damping due to the lower coil turns and smaller magnets used. This can be seen from Figs. 5 and 6. Furthermore, the graph in Fig. 5 shows that the power is a maximum for the value of load resistance at which electromagnetic damping and parasitic damping are equal. For generator B, the electromagnetic damping is always much less than the parasitic damping and the power is maximized for a load resistance equal to the coil resistance.

\section{CONCLUSION}

The model for an electromagnetic-based vibrational energy harvesting device has been compared to measurements on real devices in order to verify the modeling approach. The

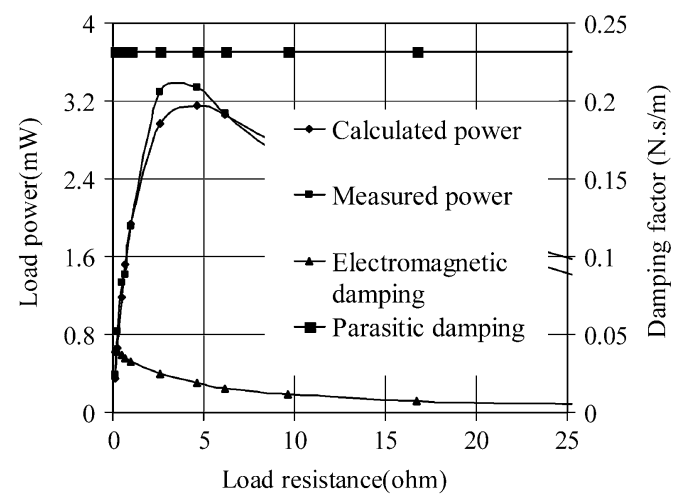

Fig. 6. Measured and calculated load power and estimated parasitic and electromagnetic damping for generator B.

optimum conditions observed from measurements are shown to agree well with the model. However, the experimental results suggest that significant parasitic damping is always present. This parasitic damping is likely to be much more of a problem for generators fabricated on the micro-scale, as the ability to achieve high electromagnetic damping reduces significantly with size [9], thus, making it difficult to achieve the optimum condition of electromagnetic damping equal to parasitic damping.

\section{ACKNOWLEDGMENT}

This work was supported in part by the European Union Framework 6 Project VIBES under Project 507911 and by the Higher Education Authority of Ireland Fund for Digital Research.

\section{REFERENCES}

[1] W. J. Li, T. C. H. Ho, G. M. H. Chan, P. H. W. Leong, and H. Y. Wong, "Infrared signal transmission by a laser-micromachined vibration-induced power generator," in 43rd IEEE Symp. Circuits Syst., 2000, pp. 236-239.

[2] C. B. Williams, C. Shearwood, M. A. Harradine, P. H. Mellor, T. S. Birch, and R. B. Yates, "Development of an electromagnetic micro-generator," Int. Elect. Eng. Proc. -Circuits Devices Syst., vol. 148, no. 6, pp. 337-342, Dec. 2001.

[3] M. El-hami, P. Glynne-Jones, N. M. White, M. Hill, S. Beeby, E. James, A. D. Brown, and J. N. Ross, "Design and fabrication of a new vibrationbased electromechanical power generator," Sens. Actuators A, vol. 92, pp. 335-342, 2001.

[4] P. Glynne-Jones, M. J. Tudor, S. P. Beeby, and N. M. White, "An electromagnetic, vibration-powered generator for intelligent sensor systems," Sens. Actuators, no. 1-3, pp. 344-349, Feb. 2003.

[5] S. Roundy and P. K. Wright, "A piezoelectric vibration based generator for wireless electronics," Smart Mater. Structures, vol. 13, pp. 1131-1142, 2004.

[6] P. D. Mitcheson, T. C. Green, E. M. Yeatman, and A. S. Holmes, "Architectures for vibration-driven micropower generators," J. Microelectromech. Syst., vol. 13, no. 3, Jun. 2004.

[7] S. P. Beeby, M. J. Tudor, E. Koukharenko, T. O’Donnell, C. Saha, S. Kulkarni, and S. Roy, "Micromachined silicon generator for harvesting power from vibrations," in Proc. Power MEMS, 2004, pp. 28-30.

[8] S. P. Beeby, M. J. Tudor, E. Koukharenko, N. M. White, T. O'Donnell, C. Saha, S. Kulkarni, and S. Roy, "Design and performance of a microelectromagnetic vibration powered generator," Transducers'05, vol. 1, pp. 780-783, Jun. 2005.

[9] T. O'Donnell, C. Saha, S. Beeby, and J. Tudor, "Scaling effects for electromagnetic vibrational power generators," in Proc. DTIP, 2006, pp. $25-28$.

Manuscript received March 13, 2006 (e-mail: chitta@tyndall.ie). 